\title{
THE GROUP GENERATED BY RIORDAN INVOLUTIONS.
}

\author{
ANA LUZÓN*, MANUEL A. MORÓN AND L. FELIPE PRIETO-MARTINEZ†
}

\begin{abstract}
We prove that any element in the group generated by the Riordan involutions is the product of at most four of them. We also give a description of this subgroup as a semidirect product of a special subgroup of the commutator subgroup and the Klein four-group.
\end{abstract}

Keywords: Riordan groups, product of involutions, commutator subgroup, inverse limit. MSC: 20H20, 15B99.

\section{INTRODUCTION}

To set our results in a wider context, we have to say that the group generated by the involutions in a group $G$ has been studied for different kinds of groups. In many of these groups has been obtained that any element in the group generated by the involutions in $G$ is the product of at most four of them. See as a sample [20, 13, 48, 11, 12, 21, 22, 36, 44, 37, 34] and the references therein. The aim of this paper is to prove the same result for the Riordan groups $\mathcal{R}(\mathbb{K})$ and $\mathcal{R}_{n}(\mathbb{K}), n \geq 1$. That is,

Theorem 1. Let $n \geq 1$ and $G=\mathcal{R}(\mathbb{K})$ or $\mathcal{R}_{n}(\mathbb{K})$. Then, any element in the group generated by the involutions in $G$ is the product of at most four of them.

The Riordan group $\mathcal{R}(\mathbb{K})$ is a multiplicative group whose elements are some special infinite lower triangular matrices with entries in $\mathbb{K}$, a field of characteristic zero. Some of its subgroups and many of its elements appear in many different context along the time. For instance in Rota's and collaborators' description of Umbral Calculus or equivalently the study of Sheffer sequences of polynomials [40], see also [3, 19, 15, 47, 30, 31. Even more, every generalized Appell polynomial sequence [6] can be obtained by means of a Riordan matrix. In particular, the matrices associated to many classical polynomial sequences are Riordan matrices. For example: Chebyshev polynomials, Fibonacci polynomials, Pell polynomials, Morgan-Voyce polynomials, Fermat polynomials. See [30]. Of course, the sequence of binomial polynomials gives rise to the oldest and most studied Riordan matrix: Pascal's triangle.

The group of invertible infinite lower triangular Toeplitz matrices is a subgroup of the Riordan group. Also, the family of all invertible Jabotinsky matrices. These matrices can be considered as matrices associated to composition operators in suitably chosen spaces. 
Riordan matrices correspond to invertible weighted composition operators in an appropriate space. See [28]. The above observation describes the way a Riordan matrix transforms power series. The formula representing this transformation is called the Fundamental Theorem of Riordan Arrays by some authors in the specific literature. The group of the formal power series of order one with composition and the so-called substitution group of formal power series are naturally isomorphic to some subgroups of the Riordan group. See for example [5, 16, 17, 41, 36, 2]. Verde-Star in [46] describes a group of operators in the multivariate context that now can be interpreted as an extension of the Riordan group in several variables. See [7, 38] for some recent related results.

The Riordan group was introduced, under this name and in a more restrictive context, by L. Shapiro and collaborators in [42. Soon after R. Sprugnoli [45] obtained many combinatorial identities using this group. Because of the origin of Riordan arrays, they are intrinsically related to Combinatorics. There, there are its first and most of its applications. Currently, the Riordan group is being studied under different angles, including the algebraic structure, the construction of new matrices from old, polynomials associated, Riordan pattern quest, among some others. A non-exhaustive list of works related to Riordan group is the following [4, 8, 9, 14, 18, 25, 26, 33, 35, 39, 43, 47, 49] and references therein. The first and second authors ran into this group from a fixed point problem to compute the quotient of series $\frac{f}{g}$, see $[28,29,24]$. This caused the $T(f \mid g)$ notation for a Riordan matrix.

In [27] $\mathcal{R}(\mathbb{K})$ is described as an inverse limit of a certain inverse sequence of groups $\mathcal{R}_{n}(\mathbb{K})$ formed by $(n+1) \times(n+1)$ invertible lower triangular matrices. For $\mathbb{K}=\mathbb{R}$ or $\mathbb{C}$, the groups $\mathcal{R}_{n}(\mathbb{K})$ have a natural structure of Lie groups. Using both facts above, in [10] the authors got and exploited a Frechet-Lie group structure in $\mathcal{R}(\mathbb{K})$. Another consequence of the inverse limit approach is the description of any involutions in every Riordan group $\mathcal{R}_{n}(\mathbb{K})$ and $\mathcal{R}(\mathbb{K})$, see [32]. This is the starting point for this paper.

In Section 2 we recall some basic facts needed about Riordan matrices, specially those related to involutions in Riordan groups. In Section 3 we observe that the subgroup of Riordan matrices with ones in the main diagonal plays a significant role in our context. In fact, we prove that this subgroup is the commutator subgroup and that any of its elements is a commutator. We illustrate our result with Pascal's triangle example. Section 4 contains the main results in this paper. In particular, a detailed proof of Theorem 1, a description of the group generated by involutions involving the semidirect product of a special subgroup of the commutator subgroup and a copy of the Klein four-group. Finally, we compute the minimal number of involutions needed to describe any element in the group generated by them in $\mathcal{R}_{n}, n \geq 1$ and in $\mathcal{R}$. 
To end this introduction, we would like to note that Riordan groups, far from being finite and/or simple, share with finite non-abelian simple groups the commutator and involution width. See the recent crucial papers [23] and [34].

\section{Previous Results.}

In this paper $\mathbb{N}$ represents the set $\{0,1,2,3, \cdots\} \subset \mathbb{K}$. An element $D=\left(d_{i, j}\right)_{i, j \in \mathbb{N}}$ in the Riordan group $\mathcal{R}(\mathbb{K})$ denoted by $(d, h)$ or $T(f \mid g)$ is an infinite matrix whose entries are $d_{i, j}=\left[x^{i}\right] d(x) h^{j}(x)$ for $(d, h)$ notation or $d_{i, j}=\left[x^{i}\right] \frac{x^{j} f(x)}{g^{j+1}(x)}$ for $T(f \mid g)$ notation, with $d, f, g \in \mathbb{K}[[x]]$ invertible for Cauchy product and $h \in \mathbb{K}[[x]]$ invertible for composition operation. Moreover, $\left[x^{k}\right]$ denotes the $k$-th coefficient in the series expansion. Note that, by definition, these matrices are invertible infinite lower triangular. In terms of the parameters the operations in the group are:

$$
(d, h)(l, m)=(d l(h), m(h)), \quad(d, h)^{-1}=\left(\frac{1}{d\left(h^{-1}\right)}, h^{-1}\right)
$$

where $h^{-1} \circ h=h \circ h^{-1}=x$. Or

$$
T(f \mid g) T(r \mid s)=T\left(f r\left(\frac{x}{g}\right) \mid g s\left(\frac{x}{g}\right)\right), \quad T^{-1}(f \mid g)=T\left(\frac{1}{f\left(\frac{x}{A}\right)} \mid A\right)
$$

where $\frac{x}{g} \circ \frac{x}{A}=\frac{x}{A} \circ \frac{x}{g}=x$ and $\alpha \beta\left(\frac{x}{\gamma}\right)$ means $\alpha(x) \beta\left(\frac{x}{\gamma(x)}\right)$. The series $A$ is the so-called $A$ sequence associated to the Riordan matrix $T(f \mid g)$. The $A$-sequence allows us to construct horizontally, i. e. by rows, in the following way

$$
d_{i, j}=\sum_{k=0}^{i-j} a_{k} d_{i-1, j-1+k} \quad i, j \geq 1
$$

where $A=\sum_{n \geq 0} a_{n} x^{n}$. See [39]. Note that the series $g=\sum_{n \geq 0} g_{n} x^{n}$ allows us to construct vertically, i.e. by columns, a Riordan matrix in the following way

$$
d_{i, j}=\sum_{k=0}^{i-j} g_{k} d_{i+1-k, j+1} \quad i, j \geq 0 .
$$

See [28, 24]. In the special case that $D=T(f \mid g)$ is a Riordan involution, then $A=g$. In fact, $\mathrm{g}$ is always the $A$-sequence of the inverse of $D$. See Proposition 7 in [24].

The action induced by $D=(d, h)$ in $\mathbb{K}[[x]]$ is given by

$$
(d, h) \alpha=d \alpha(h) \text { for } \alpha \in \mathbb{K}[[x]] .
$$

That is, $(d, h)$ is a weighted composition operator in $\mathbb{K}[[x]]$. One can get consistently the corresponding formula for the $T(f \mid g)$ notation. 
For every $n \in \mathbb{N}$ consider the general linear group $G L(n+1, \mathbb{K})$ formed by all $(n+$ $1) \times(n+1)$ invertible matrices with coefficients in $\mathbb{K}$. In the sequel, if it cause not confusion, we denote only by $\mathcal{R}$ or $\mathcal{R}_{n}$ to refer to Riordan groups. Since every Riordan matrix is lower triangular, we can define a natural homomorphism $\Pi_{n}: \mathcal{R} \rightarrow$ $G L(n+1, \mathbb{K})$ given by $\Pi_{n}\left(\left(d_{i, j}\right)_{i, j \in \mathbb{N}}\right)=\left(d_{i, j}\right)_{i, j=0,1, \cdots, n}$. Consider the subgroup $\mathcal{R}_{n}=\Pi_{n}(\mathcal{R})$ of $G L(n+1, \mathbb{K})$. We can recover the group $\mathcal{R}$ as the inverse limit of the inverse sequence of groups $\left\{\left(\mathcal{R}_{n}\right)_{n \in \mathbb{N}},\left(P_{n}\right)_{n \in \mathbb{N}}\right\}$ where $P_{n}: \mathcal{R}_{n+1} \rightarrow \mathcal{R}_{n}$ is such that if $D \in \mathcal{R}_{n+1}, P_{n}(D)$ is obtained from $D$ by deleting its last row and its last column, i.e. $P_{n}\left(\left(d_{i, j}\right)_{i, j=0,1, \cdots, n+1}\right)=$ $\left(d_{i, j}\right)_{i, j=0,1, \cdots, n}$. See [27]. Obviously if $n=0$ then $\mathcal{R}_{0}=\mathbb{K}^{*}$ with the usual product in $\mathbb{K}$ being $\mathbb{K}^{*}=\mathbb{K} \backslash\{0\}$.

Later in [32, using the inverse limit approach described above we got

Theorem 2. (Riordan Involution's Formula) Suppose $n \geq 2$. Let $D=\left(d_{i, j}\right) \in \mathcal{R}_{n-1}$ be an involution and take $\hat{D}=\left(d_{i, j}\right) \in \mathcal{R}_{n}$ such that $P_{n-1}(\hat{D})=D$.

(a) If $n$ is even, $\hat{D}$ is an involution if and only if $d_{n, 1}$ is arbitrary and

$$
d_{n, 0}=-\frac{1}{2 d_{0,0}} \sum_{k=1}^{n-1} d_{n, k} d_{k, 0}
$$

(b) If $n$ is odd, $\hat{D}$ is an involution if and only if $d_{n, 0}$ is arbitrary and

$$
d_{n, 1}=-\frac{1}{2 d_{1,1}} \sum_{k=2}^{n-1} d_{n, k} d_{k, 1}
$$

Moreover, if $a_{0}, \cdots, a_{n-2}$ are the parameters in Theorem 5 in [27] to construct $D$, then the needed $a_{n-1}$ to construct $\hat{D}$ is given by the formula

$$
a_{n-1}=\frac{1}{d_{n-1, n-1}}\left(d_{n, 1}-\sum_{j=0}^{n-2} a_{j} d_{n-1, j}\right)
$$

A consequence of Theorem 2 is the following.

Corollary 3. Let $\alpha=\sum_{i \in \mathbb{N}} \alpha_{i} x^{i}$ be an arbitrary formal power series then

(i) There is an unique nontrivial involution $D=\left(d_{i, j}\right)_{i, j \in \mathbb{N}}$ such that

$d_{0,0}=1, \quad d_{2 i+1,0}=\alpha_{2 i} \quad$ and $\quad d_{2 i+2,1}=\alpha_{2 i+1} \quad$ for $\quad i=0,1, \cdots$ we denote it by $\mathcal{I}_{\alpha}^{+}$.

(ii) There is an unique nontrivial involution $D=\left(d_{i, j}\right)_{i, j \in \mathbb{N}}$ such that

$d_{0,0}=-1, \quad d_{2 i+1,0}=\alpha_{2 i} \quad$ and $\quad d_{2 i+2,1}=\alpha_{2 i+1} \quad$ for $\quad i=0,1, \cdots$ we denote it by $\mathcal{I}_{\alpha}^{-}$. 
Moreover, any nontrivial Riordan involution can be constructed by this way.

The next two propositions were also obtained in [32]. $T(f \mid g)$ notation seems to be specially adequate to obtain them.

Proposition 4. If $T(f \mid g)$ is a Riordan involution then $g_{2}=0$, where $g=\sum_{n \geq 0} g_{n} x^{n}$.

Proposition 5. If $\Omega_{0}=\left\{T(f \mid g) \in \mathcal{R}, \mid g_{2}=0\right\}$, then $\Omega_{0}$ is a subgroup of $\mathcal{R}$.

Remark 6. The group $\Omega_{0}$ above can be described as the set of Riordan matrices whose $A$-sequences have null quadratic coefficient, because if $T(f \mid g)$ is an involution and $A$ its $A$-sequence then $g=A$. On the other hand, the condition $g_{2}=0$ turns into

$$
h_{2}^{2}=h_{1} h_{3}
$$

in the $(d, h)$ notation, for $h=\sum_{n \geq 1} h_{n} x^{n}$.

It is no difficult to prove that $\Omega_{0}$ is not normal in the Riordan group.

\section{The COMmutator subgroup And some Relations With involutions.}

Let $\mathfrak{I}$ be the set of all Riordan involutions and denote by $\langle\mathfrak{I}\rangle$ the group generated by $\mathfrak{I}$. To describe $\langle\mathfrak{I}\rangle$ some observations are relevant. First, note that every Riordan matrix $(d, h) \in \mathcal{R}$ can be written as the product of a Riordan matrix with 1's in the main diagonal and a diagonal Riordan matrix as follows

$$
(d, h) \in \mathcal{R}, \quad(d, h)=\left(\frac{d}{d_{0}}, \frac{h}{h_{1}}\right)\left(d_{0}, h_{1} x\right) .
$$

Second, every product of Riordan involutions has in its main diagonal 1's or -1's or alternatively 1 and -1 starting at 1 or at -1 . So, in the case that $(d, h) \in<\mathfrak{I}>$ we get that $\left(d_{0}, h_{1} x\right) \in \mathcal{K}$ where $\mathcal{K}=\left\{I,-I, \mathcal{I}_{0}^{+}, \mathcal{I}_{0}^{-}\right\}$. Moreover, $\mathcal{K}$ is a subgroup of the Riordan group (the Klein four-group), $\mathcal{K} \approx \mathbb{Z}_{2} \times \mathbb{Z}_{2}$. Third, from Proposition 20 and Proposition 21 in [32] we obtain that $\left\langle\mathfrak{I}>\leq \Omega_{0}\right.$. Finally, the matrix $\left(\frac{d}{d_{0}}, \frac{h}{h_{1}}\right)$ has 1 's in its main diagonal and if $(d, h) \in<\mathfrak{I}>$ then $\left(\frac{d}{d_{0}}, \frac{h}{h_{1}}\right) \in \Omega_{0}$.

As a summary,

(i) $\left(\frac{d}{d_{0}}, \frac{h}{h_{1}}\right)$ has 1's in its main diagonal.

(ii) If $(d, h) \in<\mathfrak{I}>$ then $\left(\frac{d}{d_{0}}, \frac{h}{h_{1}}\right) \in \Omega_{0}$ and $\left(d_{0}, h_{1} x\right) \in \mathcal{K}$.

(iii) $<\mathfrak{I}>\unlhd \Omega_{0}$.

In view of the above observations, Riordan matrices with 1's in the main diagonal play an important role. In fact, 
Theorem 7. The commutator subgroup of $\mathcal{R}$, denoted by $[\mathcal{R}, \mathcal{R}]$, is formed by all Riordan matrices with 1's in the main diagonal. That is,

$$
[\mathcal{R}, \mathcal{R}]=\left\{(d, h) \in \mathcal{R}, / d_{0}=1, h_{1}=1\right\} .
$$

Moreover, every element in $[\mathcal{R}, \mathcal{R}]$ is a commutator.

Proof. Consider the set

$$
\mathrm{C}=\left\{(d, h) \in \mathcal{R}, / d_{0}=1, h_{1}=1\right\} .
$$

Obviously, $\mathrm{C}$ is a subgroup of $\mathcal{R}$. If $D \in[\mathcal{R}, \mathcal{R}] \Rightarrow \exists C_{1}, C_{2}, \cdots, C_{k}$ commutators such that $D=C_{1} C_{2} \cdots C_{k}$. But all $C_{i}$ 's are commutators and triangular matrices then $C_{i} \in \mathrm{C}$, so $D \in \mathrm{C}$. Consequently $[\mathcal{R}, \mathcal{R}] \subseteq \mathrm{C}$.

If $D \in \mathrm{C}$ then $D=(d, h)$ with $d_{0}=1$ and $h_{1}=1$. Let $r \in \mathbb{K}$ such that $r \neq 0, r^{n} \neq$ $1, \forall n \geq 1$. Consider the diagonal Riordan matrix $A_{r}=(1, r x)$. We are going to prove that there exists at least a Riordan matrix $B_{r}=(l, m)$ depending on $r$, and of course on $D$, such that $D=\left[A_{r}, B_{r}\right]$. That is,

$$
(d, h)=(1, r x)(l, m)\left(1, \frac{x}{r}\right)\left(\frac{1}{l\left(m^{-1}\right)}, m^{-1}\right) .
$$

Using the product in the Riordan group we get

$$
(d, h)=\left(\frac{l(r x)}{l\left(m^{-1}\left(\frac{m(r x)}{r}\right)\right)}, m^{-1}\left(\frac{m(r x)}{r}\right)\right)
$$

hence

$$
\begin{gathered}
h=m^{-1}\left(\frac{m(r x)}{r}\right) \quad \text { and } \quad d=\frac{l(r x)}{l\left(m^{-1}\left(\frac{m(r x)}{r}\right)\right)} \\
h=m^{-1}\left(\frac{m(r x)}{r}\right) \Leftrightarrow m(h)=\frac{m(r x)}{r} \Leftrightarrow(1, h) m=\left(\frac{1}{r}, r x\right) m
\end{gathered}
$$

Consequently for $n \geq 2$

$$
m_{n}=\frac{1}{r^{n-1}-1} \sum_{k=1}^{n-1}\left[x^{n}\right] h^{k} m_{k}
$$

with $m_{1} \neq 0$ arbitrary and $\left[x^{n}\right] h^{k}$ represents the coefficient of $x^{n}$ of the $k$-th of the formal power series $h$. Analogously,

$$
\begin{gathered}
d=\frac{l(r x)}{l\left(m^{-1}\left(\frac{m(r x)}{r}\right)\right)} \Leftrightarrow d=\frac{l(r x)}{l(h)} \Leftrightarrow \\
\Leftrightarrow d l(h)=l(r x) \underset{6}{\Leftrightarrow}(d, h) l=(1, r x) l
\end{gathered}
$$


then $l_{0} \neq 0$ and

$$
l_{n}=\frac{1}{r^{n}-1} \sum_{k=0}^{n-1} d_{n, k} l_{k}, \quad \text { for } n \geq 1
$$

Hence $D$ is a commutator and of course $D \in[\mathcal{R}, \mathcal{R}]$.

Remark 8. Note that we have actually proved something stronger than the statement of the theorem because we can use the same matrix $A_{r}$ for any commutator.

Example 9. In view of the above result, Pascal's triangle $\left(\frac{1}{1-x}, \frac{x}{1-x}\right)$ is a commutator in the Riordan group. For example for $r \neq 0,1$ we get

$$
\left(\frac{1}{1-x}, \frac{x}{1-x}\right)=(1, r x)\left(\frac{r-1}{r-1-x}, \frac{r x-x}{r-1-x}\right)\left(1, \frac{x}{r}\right)\left(\frac{r-1}{r-1+x}, \frac{r x-x}{r-1+x}\right) .
$$

Since the abelianization of a group $G$ is the quotient group $G /[G, G]$ it is clear that

Corollary 10. The abelianization of the Riordan group is isomorphic to the subgroup formed by all diagonal Riordan matrices. Consequently, it is isomorphic to the direct product $\mathbb{K}^{*} \times \mathbb{K}^{*}$.

Note also that, in this case:

$$
\mathcal{R} \approx[\mathcal{R}, \mathcal{R}] \rtimes \mathcal{R} /[\mathcal{R}, \mathcal{R}]
$$

Remark 11. For $\mathcal{R}_{n}$ (Riordan groups of finite matrices) analogous results hold. $\left[\mathcal{R}_{n}, \mathcal{R}_{n}\right]$ is formed by all $(n+1) \times(n+1)$ Riordan matrices with 1's in the main diagonal. Moreover, for $n \geq 1$ the abelianization of $\mathcal{R}_{n}$ is also isomorphic to $\mathbb{K}^{*} \times \mathbb{K}^{*}$. Note that the group $\mathcal{R}_{0}$ is $\left(\mathbb{K}^{\star}, \cdot\right)$ and then it is abelian. In the remaining cases we obtain

$$
\mathcal{R}_{n} \approx\left[\mathcal{R}_{n}, \mathcal{R}_{n}\right] \rtimes \mathcal{R}_{n} /\left[\mathcal{R}_{n}, \mathcal{R}_{n}\right]
$$

\section{The Group Generated By Riordan involutions}

To prove Theorem 1 we have to use the following partial fact that describes how certain Riordan matrices can be expressed as product of three Riordan involutions. We are going to construct the three involutions doing an exhaustive use of the Riordan involution's Formula in Theorem 2, Theorem 12 below is the key to obtain the general result.

Theorem 12. Let $d=\sum_{n \geq 0} d_{n} x^{n}$ and $h=\sum_{n \geq 1} h_{n} x^{n}$ be two power series such that $d_{0}=1$ and $h_{1}=-1$. Suppose also that the Riordan matrix $(d, h) \in \Omega_{0}$, then there are three Riordan involutions, $\mathcal{I}_{\alpha}^{+}=\left(\delta_{1}, \omega_{1}\right), \mathcal{I}_{\beta}^{+}=\left(\delta_{2}, \omega_{2}\right), \mathcal{I}_{\gamma}^{+}=\left(\delta_{3}, \omega_{3}\right)$ such that $(d, h)=$ $\left(\delta_{1}, \omega_{1}\right)\left(\delta_{2}, \omega_{2}\right)\left(\delta_{3}, \omega_{3}\right)$ 
Proof. To prove this theorem is equivalent to show that the system of functional equations

$$
\begin{gathered}
d(x)=\delta_{1}(x) \delta_{2}\left(\omega_{1}(x)\right) \delta_{3}\left(\omega_{2}\left(\omega_{1}(x)\right)\right) \\
h(x)=\omega_{3}\left(\omega_{2}\left(\omega_{1}(x)\right)\right)
\end{gathered}
$$

has solutions with $\mathcal{I}_{\alpha}^{+}=\left(\delta_{1}, \omega_{1}\right), \mathcal{I}_{\beta}^{+}=\left(\delta_{2}, \omega_{2}\right)$ and $\mathcal{I}_{\gamma}^{+}=\left(\delta_{3}, \omega_{3}\right)$ Riordan involutions. We begin by equation (6) . Notice that the matrices $\left(1, \omega_{1}\right),\left(1, \omega_{2}\right),\left(1, \omega_{3}\right)$ are also involutions. Equation (6) is equivalent to

$$
\left(1, \omega_{1}\right)(1, h)=\left(1, \omega_{2}\right)\left(1, \omega_{3}\right) \quad \text { and then to }\left(1, \omega_{1}\right) h=\left(1, \omega_{2}\right) \omega_{3}
$$

Suppose now

$$
\left(1, \omega_{1}\right)=\left(a_{i, j}\right)_{i, j \in \mathbb{N}}, \quad\left(1, \omega_{2}\right)=\left(b_{i, j}\right)_{i, j \in \mathbb{N}}, \quad\left(1, \omega_{3}\right)=\left(c_{i, j}\right)_{i, j \in \mathbb{N}}
$$

The equation in $\mathcal{R}_{2}$ is

$$
\left(\begin{array}{ccc}
1 & 0 & 0 \\
0 & -1 & 0 \\
0 & a_{2,1} & 1
\end{array}\right)\left(\begin{array}{c}
0 \\
-1 \\
h_{2}
\end{array}\right)=\left(\begin{array}{ccc}
1 & 0 & 0 \\
0 & -1 & 0 \\
0 & b_{2,1} & 1
\end{array}\right)\left(\begin{array}{c}
0 \\
-1 \\
c_{2,1}
\end{array}\right)
$$

The above equality is equivalent to the linear equation

$$
a_{2,1}-b_{2,1}+c_{2,1}=h_{2}
$$

that has infinite solutions because $a_{2,1}, b_{2,1}$ and $c_{2,1}$ can be choosen arbitrarily by (a) in Theorem 2 .

The equation in $\mathcal{R}_{3}$ is

$$
\left(\begin{array}{cccc}
1 & 0 & 0 & 0 \\
0 & -1 & 0 & 0 \\
0 & a_{2,1} & 1 & 0 \\
0 & -a_{2,1}^{2} & -2 a_{2,1} & -1
\end{array}\right)\left(\begin{array}{c}
0 \\
-1 \\
h_{2} \\
h_{3}
\end{array}\right)=\left(\begin{array}{cccc}
1 & 0 & 0 & 0 \\
0 & -1 & 0 & 0 \\
0 & b_{2,1} & 1 & 0 \\
0 & -b_{2,1}^{2} & -2 b_{2,1} & -1
\end{array}\right)\left(\begin{array}{c}
0 \\
-1 \\
c_{2,1} \\
-c_{2,1}^{2}
\end{array}\right)
$$

The above equality is equivalent to the system

$$
\left\{\begin{array}{l}
a_{2,1}-h_{2}=b_{2,1}-c_{2,1} \\
a_{2,1}^{2}-2 h_{2} a_{2,1}-h_{3}=\left(b_{2,1}-c_{2,1}\right)^{2} .
\end{array}\right.
$$

Since $(1, h) \in \Omega_{0}$, using (4) the system above reduces to the unique linear equation (7) which is just the same as in $\mathcal{R}_{2}$. Then it has solutions.

Suppose now that the equation in $\mathcal{R}_{n}$ has solution. In $\mathcal{R}_{n+1}$ only the following new equation appears

$$
\sum_{k=1}^{n+1} a_{n+1, k} h_{k}=\sum_{k=1}^{n+1} b_{n+1, k} c_{k, 1}
$$


If $n$ is odd, (8) becomes

$$
-a_{n+1,1}+\sum_{k=2}^{n} a_{n+1, k} h_{k}+h_{n+1}=-b_{n+1,1}+\sum_{k=2}^{n} b_{n+1, k} c_{k, 1}+c_{n+1,1}
$$

that is

$$
a_{n+1,1}-b_{n+1,1}+c_{n+1,1}=h_{n+1}+\sum_{k=2}^{n}\left(a_{n+1, k} h_{k}-b_{n+1, k} c_{k, 1}\right)
$$

Induction hypothesis allows us to set solutions in $\mathcal{R}_{n}$. Once we take one of them, all in the right side of equation (9) is known by the construction of Riordan matrices by rows. Moreover, by (a) in Theorem 2, $a_{n+1,1}, b_{n+1,1}, c_{n+1,1}$ can be taken arbitrarily to construct the involutions. So, (9) has infinite solutions. The system obtained adding (9) to the previous one used to compute solutions in $\mathcal{R}_{n}$ is consistent. Then we have solutions for $n+1$.

If $n$ is even, the coefficients $a_{n+1,1}, b_{n+1,1}, c_{n+1,1}$ can not be taken arbitrarily to construct the involutions. From (2) in Theorem 2 they depend, in particular, on $a_{n, 1}, b_{n, 1}$ and $c_{n, 1}$. So, to be sure of the existence of solutions in $\mathcal{R}_{n+1}$, assuming that they exist in $\mathcal{R}_{n}$, we have to study the consistency of the system

$$
\left\{\begin{array}{l}
a_{n, 1}-b_{n, 1}+c_{n, 1}=h_{n}+\sum_{k=2}^{n-1}\left(a_{n, k} h_{k}-b_{n, k} c_{k, 1}\right) \\
\sum_{k=1}^{n+1} a_{n+1, k} h_{k}=\sum_{k=1}^{n+1} b_{n+1, k} c_{k, 1}
\end{array}\right.
$$

where the unknown variables are $a_{n, 1}, b_{n, 1}$ and $c_{n, 1}$. Using (2) in Theorem 2 we get

$$
\begin{aligned}
a_{n+1,1}=\frac{1}{2} \sum_{k=2}^{n} a_{n+1, k} a_{k, 1} & =\frac{1}{2}\left(a_{n+1,2} a_{2,1}+a_{n+1, n} a_{n, 1}\right)+K_{1} \\
b_{n+1,1}=\frac{1}{2} \sum_{k=2}^{n} b_{n+1, k} b_{k, 1} & =\frac{1}{2}\left(b_{n+1,2} b_{2,1}+b_{n+1, n} b_{n, 1}\right)+K_{2} \\
c_{n+1,1}=\frac{1}{2} \sum_{k=2}^{n} c_{n+1, k} c_{k, 1} & =\frac{1}{2}\left(c_{n+1,2} c_{2,1}+c_{n+1, n} c_{n, 1}\right)+K_{3}
\end{aligned}
$$

where

$$
K_{1}=\frac{1}{2} \sum_{k=3}^{n-1} a_{n+1, k} a_{k, 1}, \quad K_{2}=\frac{1}{2} \sum_{k=3}^{n-1} b_{n+1, k} b_{k, 1}, \quad K_{3}=\frac{1}{2} \sum_{k=3}^{n-1} c_{n+1, k} c_{k, 1} .
$$

Note that $K_{1}, K_{2}, K_{3}$ can be computed once one set a solution in $\mathcal{R}_{n-1}$ by induction hypothesis and by the construction by rows of Riordan matrices.

Since $n$ is even we get

$$
a_{n+1,2}=\sum_{k=0}^{n-1} A_{k}^{\omega_{1}} a_{n, 1+k}=-a_{n, 1}+\sum_{k=1}^{n-2} A_{k}^{\omega_{1}} a_{n, 1+k}+A_{n-1}^{\omega_{1}}
$$


where $A_{k}^{\omega_{i}}$ is the $k$-th coefficient of the $A$-sequence, $A^{\omega_{i}}$, of the involution $\left(1, \omega_{i}\right)$ for $i=$ $1,2,3$. From (3) in Theorem 2 and recalling that $a_{n, 0}=0$ for $n \geq 1$, we obtain that

$$
A_{n-1}^{\omega_{1}}=\frac{1}{a_{n-1, n-1}}\left(a_{n, 1}-\sum_{k=0}^{n-2} A_{k}^{\omega_{1}} a_{n-1, k}\right)=-a_{n, 1}+\sum_{k=1}^{n-2} A_{k}^{\omega_{1}} a_{n-1, k}
$$

hence

$$
a_{n+1,2}=-2 a_{n, 1}+K_{4},
$$

where

$$
K_{4}=\sum_{k=1}^{n-2} A_{k}^{\omega_{1}}\left(a_{n, 1+k}+a_{n-1, k}\right)
$$

In a similar way we get

$$
b_{n+1,2}=-2 b_{n, 1}+K_{5}, \quad c_{n+1,2}=-2 c_{n, 1}+K_{6},
$$

where

$$
K_{5}=\sum_{k=1}^{n-2} A_{k}^{\omega_{2}}\left(b_{n, 1+k}+b_{n-1, k}\right), \quad K_{6}=\sum_{k=1}^{n-2} A_{k}^{\omega_{2}}\left(c_{n, 1+k}+c_{n-1, k}\right) .
$$

Now, we are going to write the equation

$$
\sum_{k=1}^{n+1} a_{n+1, k} h_{k}=\sum_{k=1}^{n+1} b_{n+1, k} c_{k, 1}
$$

as

$-a_{n+1,1}+a_{n+1,2} h_{2}+\sum_{k=3}^{n+1} a_{n+1, k} h_{k}=-b_{n+1,1}+b_{n+1,2} c_{2,1}+b_{n+1, n} c_{n, 1}-c_{n+1,1}+\sum_{k=3}^{n-1} b_{n+1, k} c_{k, 1}$

it can be written as

$$
a_{n+1,1}-b_{n+1,1}+b_{n+1,2} c_{2,1}+b_{n+1, n} c_{n, 1}-c_{n+1,1}-a_{n+1,2} h_{2}=K_{7}
$$

putting on the left side what depends on unknown variables and on the right side, $K_{7}$, what depends on the induction hypothesis and on data, note that

$$
K_{7}=\sum_{k=3}^{n+1} a_{n+1, k} h_{k}-\sum_{k=3}^{n-1} b_{n+1, k} c_{k, 1} .
$$

Moreover, as $\left(1, \omega_{i}\right) i=1,2,3$ are involutions and we are considering $n$ even, we get

$$
a_{n+1, n}=-n a_{2,1}, \quad b_{n+1, n}=-n b_{2,1}, \quad c_{n+1, n}=-n c_{2,1} .
$$

Then, gathering together every equalities above we obtain

$$
\begin{gathered}
\frac{1}{2}\left(a_{n+1,2} a_{2,1}+a_{n+1, n} a_{n, 1}\right)-\frac{1}{2}\left(b_{n+1,2} b_{2,1}+b_{n+1, n} b_{n, 1}\right)+b_{n+1,2} c_{2,1}+b_{n+1, n} c_{n, 1}- \\
-\frac{1}{2}\left(c_{n+1,2} c_{2,1}+c_{n+1, n} c_{n, 1}\right)-a_{n+1,2} h_{2}=K_{8}
\end{gathered}
$$


where $K_{8}=K_{7}-K_{1}+K_{2}+K_{3}$, o equivalently

$$
\begin{gathered}
\left(\frac{1}{2} a_{2,1}-h_{2}\right) a_{n+1,2}+\frac{1}{2} a_{n+1, n} a_{n, 1}+\left(c_{2,1}-\frac{1}{2} b_{2,1}\right) b_{n+1,2}-\frac{1}{2} b_{n+1, n} b_{n, 1}+ \\
+\left(b_{n+1, n}-\frac{1}{2} c_{n+1, n}\right) c_{n, 1}-\frac{1}{2} c_{n+1,2} c_{2,1}=K_{8}
\end{gathered}
$$

and

$$
\begin{gathered}
\left(\frac{1}{2} a_{2,1}-h_{2}\right)\left(-2 a_{n, 1}+K_{4}\right)+\frac{1}{2} a_{n+1, n} a_{n, 1}+\left(c_{2,1}-\frac{1}{2} b_{2,1}\right)\left(-2 b_{n, 1}+K_{5}\right)-\frac{1}{2} b_{n+1, n} b_{n, 1}+ \\
\left(b_{n+1, n}-\frac{1}{2} c_{n+1, n}\right) c_{n, 1}-\frac{1}{2}\left(-2 c_{n, 1}+K_{6}\right) c_{2,1}=K_{8}
\end{gathered}
$$

SO

$$
\begin{gathered}
\left(2 h_{2}-a_{2,1}+\frac{1}{2} a_{n+1, n}\right) a_{n, 1}+\left(b_{2,1}-2 c_{2,1}-\frac{1}{2} b_{n+1, n}\right) b_{n, 1}+ \\
+\left(c_{2,1}+b_{n+1, n}-\frac{1}{2} c_{n+1, n}\right) c_{n, 1}=K_{9}
\end{gathered}
$$

where

$$
K_{9}=K_{8}-\left(\frac{1}{2} a_{2,1}-h_{2}\right) K_{4}-\left(c_{2,1}-\frac{1}{2} b_{2,1}\right) K_{5}+\frac{1}{2} c_{2,1} K_{6}
$$

Finally

$$
\left(2 h_{2}-\left(\frac{n}{2}+1\right) a_{2,1}\right) a_{n, 1}+\left(\left(\frac{n}{2}+1\right) b_{2,1}-2 c_{2,1}\right) b_{n, 1}+\left(\left(\frac{n}{2}+1\right) c_{2,1}-n b_{2,1}\right) c_{n, 1}=K_{9} .
$$

It is important to note that for $m=1 \cdots 9, K_{m}$ depends only on the solution for $\mathcal{R}_{n-1}$ and on $h$.

In summary, we have to study the consistency of the linear system

$$
\left\{\begin{array}{l}
a_{n, 1}-b_{n, 1}+c_{n, 1}=h_{n}+\sum_{k=2}^{n-1}\left(a_{n, k} h_{k}-b_{n, k} c_{k, 1}\right) \\
\left(2 h_{2}-\left(\frac{n}{2}+1\right) a_{2,1}\right) a_{n, 1}+\left(\left(\frac{n}{2}+1\right) b_{2,1}-2 c_{2,1}\right) b_{n, 1}+\left(\left(\frac{n}{2}+1\right) c_{2,1}-n b_{2,1}\right) c_{n, 1}=K_{9} .
\end{array}\right.
$$

Consider the matrix

$$
\left(\begin{array}{cc}
1 & -1 \\
2 h_{2}-\left(\frac{n}{2}+1\right) a_{2,1} & \left(\frac{n}{2}+1\right) b_{2,1}-2 c_{2,1}
\end{array}\right)
$$

whose determinant is $\left(\frac{n}{2}-1\right)\left(b_{2,1}-a_{2,1}\right)$ by (17). Since we can choose $a_{2,1} \neq b_{2,1}$ in (7) and $n \geq 4$ the system is consistent and we get solutions for $n+1$.

To complete the proof of the theorem we are going to substitute in (5) the solutions found before for (6) . The involutions $\left(1, \omega_{i}\right)$ and $\left(\delta_{i}, \omega_{i}\right)$ share the $A$-sequence denoted before by $A^{\omega_{i}}$. In particular, we have to recall that $A_{1}^{\omega_{1}}=-a_{2,1}, A_{1}^{\omega_{2}}=-b_{2,1}$ and $a_{2,1} \neq b_{2,1}$ where $\left(1, \omega_{1}\right)=\left(a_{i, j}\right)$ and $\left(1, \omega_{2}\right)=\left(b_{i, j}\right)$. Additionally, we will prove also that we can always 
suppose that $\delta_{3} \equiv 1$ in (5). Doing this (5) turns into any of the following three equivalent equations

$$
\begin{gathered}
\delta_{1}(x) \delta_{2}\left(\omega_{1}(x)\right)=d(x) \\
\left(\delta_{1}, \omega_{1}\right)\left(\delta_{2}, \omega_{2}\right)(1)=d(x)
\end{gathered}
$$

or

$$
\left(\delta_{1}, \omega_{1}\right) d(x)=\left(\delta_{2}, \omega_{2}\right)(1)
$$

Suppose that

$$
\left(\delta_{1}, \omega_{1}\right)=\left(u_{i, j}\right)_{i, j \in \mathbb{N}}, \quad\left(\delta_{2}, \omega_{2}\right)=\left(v_{i, j}\right)_{i, j \in \mathbb{N}}, \quad d(x)=\sum_{k \geq 0} d_{k, 0} x^{k} .
$$

So, in $\mathcal{R}_{0}$ equation (10) holds tautologically. In $\mathcal{R}_{1}$ the equation (10) is

$$
\left(\begin{array}{cc}
1 & 0 \\
u_{1,0} & -1
\end{array}\right)\left(\begin{array}{c}
1 \\
d_{1,0}
\end{array}\right)=\left(\begin{array}{cc}
1 & 0 \\
v_{1,0} & -1
\end{array}\right)\left(\begin{array}{l}
1 \\
0
\end{array}\right)
$$

and the linear equation

$$
u_{1,0}-v_{1,0}=d_{1,0}
$$

has infinite solutions for any $d_{1,0}$ for the unknowns $u_{1,0}$ and $v_{1,0}$ and then we have solutions in $\mathcal{R}_{1}$. In $\mathcal{R}_{2}$ the equation (10) is

$$
\left(\begin{array}{ccc}
1 & 0 & 0 \\
u_{1,0} & -1 & 0 \\
-\frac{1}{2} u_{1,0} u_{2,1} & u_{2,1} & 1
\end{array}\right)\left(\begin{array}{c}
1 \\
d_{1,0} \\
d_{2,0}
\end{array}\right)=\left(\begin{array}{ccc}
1 & 0 & 0 \\
v_{1,0} & -1 & 0 \\
-\frac{1}{2} v_{1,0} v_{2,1} & v_{2,1} & 1
\end{array}\right)\left(\begin{array}{l}
1 \\
0 \\
0
\end{array}\right)
$$

then we have to solve the system whose first equation is (11) and the second one is

$$
-\frac{1}{2} u_{1,0} u_{2,1}+u_{2,1} d_{1,0}+d_{2,0}=-\frac{1}{2} v_{1,0} v_{2,1}
$$

or equivalently

$$
\left(d_{1,0}-\frac{1}{2} u_{1,0}\right) u_{2,1}+d_{2,0}=-\frac{1}{2} v_{1,0} v_{2,1}
$$

Using (11) we get

$$
\left(v_{1,0}-\frac{1}{2} u_{1,0}\right) u_{2,1}-\frac{1}{2} v_{1,0} v_{2,1}=d_{2,0} .
$$

By the construction of Riordan matrices by means of the A-sequence we obtain

$$
u_{2,1}=a_{2,1}-u_{1,0}, \quad v_{2,1}=b_{2,1}-v_{1,0},
$$

consequently

$$
\left(v_{1,0}-\frac{1}{2} u_{1,0}\right)\left(a_{2,1}-u_{1,0}\right)-\frac{1}{2} v_{1,0}\left(b_{2,1}-v_{1,0}\right)=d_{2,0}
$$


or

$$
\frac{1}{2}\left(u_{1,0}-v_{1,0}\right)^{2}+\left(v_{1,0}-\frac{1}{2} u_{1,0}\right) a_{2,1}-\frac{1}{2} v_{1,0} b_{2,1}=d_{2,0} .
$$

Doing some computations we obtain

$$
-a_{2,1} u_{1,0}+\left(2 a_{2,1}-b_{2,1}\right) v_{1,0}=2 d_{2,0}-d_{1,0}^{2}
$$

So, the linear system to solve is

$$
\left\{\begin{array}{l}
u_{1,0}-v_{1,0}=d_{1,0}, \\
-a_{2,1} u_{1,0}+\left(2 a_{2,1}-b_{2,1}\right) v_{1,0}=2 d_{2,0}-d_{1,0}^{2} .
\end{array}\right.
$$

Using (17) and (11) we prove that the above system has solutions. Consequently we solve our problem for $\mathcal{R}_{2}$.

We proceed by induction in a similar way to the previous case. Suppose we have solved (5) in $\mathcal{R}_{n}$ and we want to solve it in $\mathcal{R}_{n+1}$. Then we have the linear system with the $n$ previous equations and the new equation

$$
\sum_{j=0}^{n+1} u_{n+1, j} d_{j, 0}=v_{n+1,0}
$$

which is the same as

$$
u_{n+1,0}-v_{n+1,0}=d_{n+1,0}-\sum_{j=1}^{n} u_{n+1, j} d_{j, 0}
$$

or

$$
u_{n+1,0}-v_{n+1,0}=L_{1}
$$

with $L_{1}=d_{n+1,0}-\sum_{j=1}^{n} u_{n+1, j} d_{j, 0}$. As in the case of the previous symbols $K_{m}, L_{j}$ groups together terms depending on induction hypothesis and data.

Since the elements $(2 k+1,0)$ in an involution are arbitrary and the elements $(2 k, 0)$ are given by (1) we have to distinguish two cases. In the case $n+1$ odd, $u_{n+1,0}$ and $v_{n+1,0}$ are arbitrary. Then, the linear system with the new equation has solutions. In the case that $n+1$ even, these elements are given by (1). Then we must study the consistency of the linear system

$$
\left\{\begin{array}{l}
u_{n, 0}-v_{n, 0}=d_{n, 0}-\sum_{j=1}^{n-1} u_{n, j} d_{j, 0}, \\
\sum_{j=0}^{n+1} u_{n+1, j} d_{j, 0}=v_{n+1,0} .
\end{array}\right.
$$

By using formula (11) we get

$$
\begin{gathered}
u_{n+1,0}=-\frac{1}{2}\left(u_{n+1,1} u_{1,0}+u_{n+1, n} u_{n, 0}\right)+L_{2} \\
v_{n+1,0}=-\frac{1}{2}\left(v_{n+1,1} v_{1,0}+v_{n+1, n} v_{n, 0}\right)+L_{3} \\
13
\end{gathered}
$$


where

$$
L_{2}=-\frac{1}{2} \sum_{k=2}^{n-1} u_{n+1, k} u_{k, 0} \quad \text { and } \quad L_{3}=-\frac{1}{2} \sum_{k=2}^{n-1} v_{n+1, k} v_{k, 0} .
$$

Moreover, by means of the horizontal construction of a Riordan matrix we have

$$
u_{n+1,1}=-u_{n, 0}+L_{4}, \quad v_{n+1,1}=-v_{n, 0}+L_{5},
$$

where

$$
L_{4}=\sum_{k=1}^{n} A_{k}^{\omega_{1}} u_{n, k}, \quad L_{5}=\sum_{k=1}^{n} A_{k}^{\omega_{2}} v_{n, k}
$$

Beside, we know that

$$
u_{n+1, n}=-u_{1,0}+n a_{2,1} \quad \text { and } \quad v_{n+1, n}=-v_{1,0}+n b_{2,1} .
$$

By (11) we can write equation (12) as

$$
u_{n+1,0}+u_{n+1,1}\left(u_{1,0}-v_{1,0}\right)-v_{n+1,0}=L_{6},
$$

where $L_{6}=-\sum_{j=2}^{n+1} u_{n+1, j} d_{j, 0}$. Now we replace the expressions above to obtain

$$
\begin{aligned}
& \left.-\frac{1}{2}\left(u_{n+1,1} u_{1,0}+u_{n+1, n} u_{n, 0}\right)+u_{n+1,1}\left(u_{1,0}-v_{1,0}\right)-\frac{1}{2}\left(v_{n+1,1} v_{1,0}+v_{n+1, n} v_{n, 0}\right)\right)=L_{6}-L_{2}+L_{3} \\
& \left.\left(\frac{1}{2} u_{1,0}-v_{1,0}\right) u_{n+1,1}-\frac{1}{2} u_{n+1, n} u_{n, 0}-\frac{1}{2}\left(v_{n+1,1} v_{1,0}+v_{n+1, n} v_{n, 0}\right)\right)=L_{6}-L_{2}+L_{3} \\
& \left.\left(\frac{1}{2} u_{1,0}-v_{1,0}\right)\left(-u_{n, 0}+L_{4}\right)-\frac{1}{2}\left(-u_{1,0}+n a_{2,1}\right) u_{n, 0}-\frac{1}{2}\left(\left(-v_{n, 0}+L_{5}\right) v_{1,0}+\left(-v_{1,0}+n b_{2,1}\right) v_{n, 0}\right)\right)=L_{6}-L_{2}+L_{3} \\
& \left.\left(\frac{1}{2} u_{1,0}-v_{1,0}\right)\left(-u_{n, 0}\right)-\frac{1}{2}\left(-u_{1,0}+n a_{2,1}\right) u_{n, 0}-\frac{1}{2}\left(\left(-v_{n, 0}\right) v_{1,0}+\left(-v_{1,0}+n b_{2,1}\right) v_{n, 0}\right)\right)=L_{7}
\end{aligned}
$$

where

$$
L_{7}=L_{6}-L_{2}+L_{3}-\left(\frac{1}{2} u_{1,0}-v_{1,0}\right) L_{4}+\frac{1}{2} v_{1,0} L_{5}
$$

reorganizing the variables we get the linear system

$$
\left\{\begin{array}{l}
u_{n, 0}-v_{n, 0}=d_{n, 0}-\sum_{j=1}^{n-1} u_{n, j} d_{j, 0} \\
\left(v_{1,0}-\frac{n}{2} a_{2,1}\right) u_{n, 0}+\left(\frac{n}{2} b_{2,1}-v_{1,0}\right) v_{n, 0}=L_{7}
\end{array}\right.
$$

It has solutions because

$$
\left|\begin{array}{cc}
1 & -1 \\
v_{1,0}-\frac{n}{2} a_{2,1} & \frac{n}{2} b_{2,1}-v_{1,0}
\end{array}\right|=\frac{n}{2} b_{2,1}-v_{1,0}+v_{1,0}-\frac{n}{2} a_{2,1}=\frac{n}{2}\left(b_{2,1}-a_{2,1}\right) \neq 0
$$

that is the needed condition for the equation (6) holds. Then the case $n+1$ has also solutions.

So, we have proved that our result is true in $\mathcal{R}_{n}$ for every $n \in \mathbb{N}$. Since the group $\mathcal{R}$ is the inverse limit of the inverse sequence of groups $\left\{\left(\mathcal{R}_{n}\right)_{n \in \mathbb{N}},\left(P_{n}\right)_{n \in \mathbb{N}}\right\}$ and $P_{n}$ transforms solutions in $\mathcal{R}_{n+1}$ to solutions in $\mathcal{R}_{n}$, the proof is finished. 
Note that, if $D \in<\mathfrak{I}>$, the main diagonal of $D$ is the same as the main diagonal of one of the diagonal Riordan involutions. In each of the cases, we can multiply $D$ by one of the diagonal involutions to get a Riordan matrix $(d, h)$ with $d_{0}=1$ and $h_{1}=-1$, then by Theorem 12 we obtain that $D$ is a product of at most four involutions. Then we have proved Theorem 1 for $\mathcal{R}$ and $\mathcal{R}_{n}, n \geq 1$.

In fact we can describe, up to isomorphism, the group generated by involutions using the commutator subgroup and the semidirect product concept in the following way.

\section{Theorem 13.}

$$
<\mathfrak{I}>\approx[\mathcal{R}, \mathcal{R}]_{0} \rtimes \mathcal{K}
$$

where $[\mathcal{R}, \mathcal{R}]_{0}=\Omega_{0} \cap[\mathcal{R}, \mathcal{R}]$ and $\mathcal{K}=\left\{I,-I, \mathcal{I}_{0}^{+}, \mathcal{I}_{0}^{-}\right\}$.

Proof. Note that $\langle\mathfrak{I}\rangle \subseteq[\mathcal{R}, \mathcal{R}]_{0} \mathcal{K}$ where $[\mathcal{R}, \mathcal{R}]_{0} \mathcal{K}$ represents the set of Riordan matrices obtained by multiplying an element in $[\mathcal{R}, \mathcal{R}]_{0}$ with an element in $\mathcal{K}$ in such order. Now suppose $D K \in[\mathcal{R}, \mathcal{R}]_{0} \mathcal{K}$ with $D \in[\mathcal{R}, \mathcal{R}]_{0}$ and $K \in \mathcal{K}$. Then $D \mathcal{I}_{0}^{+}=\mathcal{I}_{\alpha}^{+} \mathcal{I}_{\beta}^{+} \mathcal{I}_{\gamma}^{+}$by Theorem 12. Consequently $D=\mathcal{I}_{\alpha}^{+} \mathcal{I}_{\beta}^{+} \mathcal{I}_{\gamma}^{+} \mathcal{I}_{0}^{+}$, hence $D K \in<\mathfrak{I}>$. So, $<\mathfrak{I}>=[\mathcal{R}, \mathcal{R}]_{0} \mathcal{K}$. We also proved in Theorem 12 that, in fact, $\left.[\mathcal{R}, \mathcal{R}]_{0}=[\mathcal{R}, \mathcal{R}] \cap<\mathfrak{I}\right\rangle$. Therefore $\left.[\mathcal{R}, \mathcal{R}]_{0} \unlhd<\mathfrak{I}\right\rangle$. Finally, $[\mathcal{R}, \mathcal{R}]_{0} \cap \mathcal{K}=\{I\}$. This implies the result by using [1] page 133 .

Final remark: It is easy to prove that any element in the group generated by involutions in $\mathcal{R}_{1}$ can be described as the product of two of them. Using Theorem 2 herein and Corollary 7 in [32], the matrix

$$
\left(\begin{array}{lll}
1 & 0 & 0 \\
0 & 1 & 0 \\
1 & 0 & 1
\end{array}\right)=\left(\begin{array}{ccc}
1 & 0 & 0 \\
1 & -1 & 0 \\
-1 & 2 & 1
\end{array}\right)\left(\begin{array}{ccc}
1 & 0 & 0 \\
1 & -1 & 0 \\
0 & 0 & 1
\end{array}\right)\left(\begin{array}{ccc}
1 & 0 & 0 \\
0 & -1 & 0 \\
0 & -2 & 1
\end{array}\right)\left(\begin{array}{ccc}
1 & 0 & 0 \\
0 & -1 & 0 \\
0 & 0 & 1
\end{array}\right)
$$

points out that, for $n \geq 2$, in $\mathcal{R}_{n}$ and in $\mathcal{R}$ there are elements in the group generated by involutions that can not be described as the product of three or less involutions.

Acknowledgment: The first and second authors were partially supported by grant MINECO, MTM2015-63612-P.

\section{REFERENCES}

[1] M.A. Amstrong. Groups and Symmetry. Springer-Verlag. 1988.

[2] I.K. Babenko. Algebra, geometry and topology of the substitution group of formal power series. Russian Math. Surveys 68 (1) (2013) 1-68.

[3] M. Barnabei, A. Brini and G. Nicoletti. Recursive matrices and umbral calculus. Journal of Algebra 75 (1982) 546-573.

[4] P. Barry, A. Hennessy Meixner-type results for Riordan arrays and associated integer sequences. J. Integer Seq. 13 no 9 (2010) Article 10.9.4, 34 pp. 
[5] A. Bennett. The iterarion of functions of one variable. Ann. of Math. 17 (1) (1915) 23-60.

[6] R.P. Boas, R.C. Buck. Polynomial expansions of analytic functions. Springer-Verlag (1964.)

[7] G.-S. Cheon, I-C. Huang, S. Kim. Multivariate Riordan groups and their representations. Linear Algebra Appl. 514 (2017) 198-207.

[8] G.-S. Cheon, S.-T. Jin. Structural properties of Riordan matrices and extending the matrices. Linear Algebra Appl. 435 (2011) 2019-2032.

[9] G.-S. Cheon, H. Kim. Simple proofs of open problems about the structure of involutions in the Riordan group. Linear Algebra Appl. 428 (2008) 930-940.

[10] G.-S. Cheon, A. Luzon, M. A. Moron, L. F. Prieto-Martinez and M. Song Finite and infinite dimensional Lie group structures on Riordan groups. Adv. Math. 319 (2017) 522-566.

[11] D.Z. Djokovic. Product of two involutions. Arch. Math. Vol 18 (1967) 582-584.

[12] W. H. Gustafson, P.R. Halmos, H. Radjavi. Products of involutions. Linear Algebra Appl. Vol 13 (1976) 157-163.

[13] P.R. Halmos, S. Kakutani. Product of symmetries. Bull. Amer. Math. Soc. Vol 64, No 3 (1958) $77-78$.

[14] T-X. He, Shift operators defined in the Riordan group and their applications. Linear Algebra Appl. 496 (2016) 331-350

[15] T-X. He, L.C. Hsu, P.J-S. Shiue. The Sheffer group and the Riordan group. Discrete Applied Mathematics. 155 (2007) 1895-1909.

[16] E. Jabotinsky. Sur la representation de la composition de foncions par un produit de matrices. Application à l'itération de e $e^{z}$ et de $e^{z}-1$. C. R. Acad. Sci. Paris 224 (1947) 323-324.

[17] E. Jabotinsky. Representation of functions by matrices. Application to Faber polynomials. Proc. Amer. Math. Soc. 4 (1953) 546-553.

[18] C. Jean-Louis and A. Nkwanta. Some algebraic structure of the Riordan group. Linear Algebra Appl. 438 (2013) 2018-2035.

[19] D. E. Knuth. Convolution polynomials. The Mathematica journal. 2 (1992.) 67-78.

[20] E. Kasner. Infinite groups generated by conformal transformations of period two (involutions and symmetries). Amer. J. Math. 38 (1916) 177-184.

[21] F. Knüppel, K. Nielsen. SL(V) is 4-reflectional. Geom. Dedicata 38 (1991) 301-308.

[22] F. Knüppel, G. Thomsen. Involutions and commutators in orthogonal groups. J. Aust. Math. Soc. A 64 (1998) 1-36.

[23] M.W. Liebeck, E. A. O'Brien, A. Shalev, P. H. Tiep. The Ore conjecture. J. Eur. Math. Soc. 12 (2010) 939-1008. 
[24] A. Luzón. Iterative processes related to Riordan arrays: The reciprocation and the inversion of power series. Discrete Math. 310 (2010) 3607-3618.

[25] A. Luzón, D. Merlini, M. A. Morón and R. Sprugnoli. Identities induced by Riordan arrays. Linear Algebra Appl. 436 (2012) 631-647.

[26] A. Luzón, D. Merlini, M. A. Morón and R. Sprugnoli. Complementary Riordan arrays. Discrete Appl. Math. 172 (2014) 75-87.

[27] A. Luzón, D. Merlini, M. A. Morón, L. F. Prieto-Martínez and R. Sprugnoli. Some inverse limit approaches to the Riordan group. Linear Algebra Appl. 491 (2016) 239-262.

[28] A. Luzón and M. A. Morón. Ultrametrics, Banach's fixed point theorem and the Riordan group. Discrete Appl. Math. 156 (2008) 2620-2635.

[29] A. Luzón and M. A. Morón. Riordan matrices in the reciprocation of quadratic polynomials. Linear Algebra Appl. 430 (2009) 2254-2270.

[30] A. Luzón, M. A. Morón. Recurrence relations for polynomial sequences via Riordan matrices. Linear Algebra Appl. 433 (2010) 1422-1446.

[31] A. Luzon and M. A. Morón. Self-inverse Sheffer sequences and Riordan involutions. Discrete Appl. Math. 159 (2011) 1290-1292.

[32] A. Luzón, M. A. Morón and L. F. Prieto-Martínez. A formula to construct all involutions in Riordan matrix groups. Linear Algebra Appl. 533 (2017) 397-417.

[33] A. Luzón, M. A. Morón and J. L. Ramírez. Double parameter recurrences for polynomials in bi-infinite Riordan matrices and some derived identities. Linear Algebra Appl. 511 (2016) 237-258.

[34] A. J. Malcolm. The involution width of finite simple groups. J. Algebra 493 (2018) 297-340.

[35] D. Merlini, D. G. Rogers, R. Sprugnoli, M.C. Verri. On some alternative characterizations of Riordan arrays. Canadian J. Math. 49(2) (1997) 301-320.

[36] A. G. O'Farrell. Composition of involutive power series, and reversible series. Comput. Methods Funct. Theory 8 (2008), no. 1-2, 173-193.

[37] A.G. O'Farrell and I. Short. Reversibility in dynamics and group theory. London Mathematical Society Lecture Note Series, 416. Cambridge University Press, Cambridge, 2015. ISBN: 978-1-107-44288-7

[38] A.G. O'Farrell. Riordan groups in d dimensions: remarks. Personal communication. (2017).

[39] D.G. Rogers. Pascal triangles, Catalan numbers and renewal arrays. Discrete Math. 22 (1978) 301-310.

[40] G.C. Rota, D. Kahaner and A. Odlyzko. On the Fundations of Combinatorial Theory, VIII: Finite Operators Calculus J. Math. Anal. Appl.V. 42 (1973) 684-760 
[41] S. Scheinberg. Power series in one variable. J. Math. Anal. Appl. 31 (1970) 321-333.

[42] L. W. Shapiro, S. Getu, W.J. Woan and L. Woodson. The Riordan group. Discrete Appl. Math. 34 (1991) 229-239.

[43] L. W. Shapiro. Some open question about random walks, involutions, limiting distributions, and generating functions. Adv. Appl. Math. 27 (2001) 585-596.

[44] R. Slowik. Expressing infinite matrices as product of involutions. Linear Algebra Appl. 438 (2013) 399-404.

[45] R. Sprugnoli. Riordan arrays and combinatorial sums. Discrete Math. 132 (1994) 267-290.

[46] L. Verde-Star. Dual Operators and Lagrange Inversion in Several Variable. Adv. Math. 58 (1985) 89-108.

[47] W. Wang, T. Wang. Generalized Riordan arrays. Discrete Math. 308 (2008) 6466-6500.

[48] M. J. Wonenburger Transformations which are product of two involutions. J. Math. Mech. 16 (4) (1966) 327-338.

[49] S-L. Yang, Y-N. Dong, L. Yang, J. Yin. Half of a Riordan array and restricted lattice paths. Linear Algebra Appl. 537 (2018) 1-11.

* Departamento de Matemática Aplicada. Universidad Politécnica de Madrid (Spain).

E-mail address: anamaria.luzon@upm.es

দ Departamento de Algebra, Geometría y Topología. Universidad Complutense de Madrid and Instituto de Matemática Interdisciplinar (IMI)(Spain).

E-mail address: mamoron@mat.ucm.es

$\dagger$ IES Alpajés. Aranjuez.(Spain).

E-mail address: felipe.prieto@educa.madrid.org 